\title{
Effect of Carbon Nanotube Aqueous Dispersion Quality on Mechanical Properties of Cement Composite
}

\author{
Larisa I. Nasibulina, ${ }^{1}$ Ilya V. Anoshkin, ${ }^{1}$ Albert G. Nasibulin, ${ }^{1}$ Andrzej Cwirzen, ${ }^{2}$ \\ Vesa Penttala, ${ }^{2}$ and Esko I. Kauppinen ${ }^{1}$ \\ ${ }^{1}$ Department of Applied Physics, Aalto University School of Science, Puumiehenkuja 2, 02150 Espoo, Finland \\ ${ }^{2}$ Department of Civil and Structural Engineering, Aalto University School of Engineering, Rakentajanaukio 4 A, 02150 Espoo, Finland
}

Correspondence should be addressed to Albert G. Nasibulin, albert.nasibulin@aalto.fi

Received 24 November 2011; Accepted 6 January 2012

Academic Editor: Donglu Shi

Copyright ( 2012 Larisa I. Nasibulina et al. This is an open access article distributed under the Creative Commons Attribution License, which permits unrestricted use, distribution, and reproduction in any medium, provided the original work is properly cited.

\begin{abstract}
An effect of the quality of carbon nanotube (CNT) dispersions added to cement on paste mechanical properties has been studied. High-quality dispersions of few-walled CNT (FWCNTs) were produced in two steps. First, FWCNTs were functionalized in a mixture of nitric and sulfuric acids ( $70 \mathrm{wt} . \%$ and $96 \mathrm{wt} . \%$, resp.) at $80^{\circ} \mathrm{C}$. Second, functionalized FWCNTs were washed out by acetone to remove carboxylated carbonaceous fragments (CCFs) formed during CNT oxidation. Mechanical test results showed 2 -fold increase in the compressive strength of the cement paste prepared from the dispersion of acetone-washed functionalized FWCNTs, which is believed to occur due to the chemical interaction between cement matrix and functional groups $(-\mathrm{COOH}$ and $-\mathrm{OH})$. Utilisation of unwashed FWCNTs led to a marginal improvement of mechanical properties of the cement pastes, whereas surfactant-treated functionalized FWCNT dispersions only worsened the mechanical properties.
\end{abstract}

\section{Introduction}

Cement is one of the most commonly used construction materials due to its low cost and high workability. The world consumption of the cement is constantly growing due to rising construction engineering needs. Thus, an improvement in basic mechanical properties and durability of materials based on cement is very important. Recently, carbon nanotubes (CNTs) have attracted tremendous scientific attention owing to their excellent physical and chemical properties [1]. Due to high strength and elastic strain, CNTs were proposed to be utilized for production of cement composites with improved mechanical properties. However, usually researchers face the problem of introducing the carbon nanomaterial in the matrix, since CNTs tend to agglomerate and form bundles. CNTs have been added to the cement as a powder using rotor mixing technique $[2,3]$ or as water dispersions [4-10]. Another approach recently proposed by our group is based on direct growth of CNTs and carbon nanofibers (CNFs) on the cement particles [11, 12]. This method allowed us to achieve very good dispersion of carbon nanomaterials and, as a result, 2 -fold increase in the compressive strength of cement paste and 2.5-fold increase of mortar [13]. However, the liquid way of preparation of CNT/cement composites has not been so successful yet. The improvement in the mechanical properties of the composite materials usually did not exceed 50\% [2-10]. This fact can be explained by the quality of CNT dispersions and by the way of the dispersion preparation. The quality of the CNT water dispersions is usually judged on the basis of short-term stability without centrifugation needed to remove bundled nanotubes that can negatively affect the composite properties [14]. For getting stable CNT water dispersions one can utilize different types of surfactants. However, such kinds of dispersions are usually not able to effectively enhance mechanical properties of produced composite materials $[7,9,10]$, since CNTs are almost fully covered by polymers preventing bond formation between hydrating cement and introduced CNTs. The interaction between the cement matrix and CNTs is extremely important to reinforce cement composite and can be enhanced by creating $-\mathrm{COOH}$ and $-\mathrm{OH}$ groups on the $\mathrm{CNT}$ surface during 
acid functionalization. However, during this procedure in addition to functionalized CNTs carboxylated carbonaceous fragments (CCFs) are formed [15-17]. These fragments are organic molecules consisted of condensed aromatic graphitic rings with several functional groups. Therefore, the CCFs formed during CNT functionalization added in the cement can react with hydrating cement similarly to functionalized CNTs, but without significant effect on the mechanical properties due the absence of their structural component.

Another important question is related to the structure of the material to be added to cement, which is expected to exhibit the best composite performance. It is known that single-walled CNTs are one of the strongest materials [18]. However, after the acid functionalization they shorten and accumulate defects that negatively affected on the mechanical properties of single-walled CNTs [19]. In case of multiwalled carbon nanotubes (MWCNTs), only outer graphitic shells contribute to the strength, while inner layers practically do not participate in carrying the load and only reduce the strength per a given volume [20-22]. Young's modulus of few walled carbon nanotubes (FWCNTs) with 2-5 layers is from 0.8 to $1.6 \mathrm{TPa}$ and therefore they are believed to be the most optimum and superior candidate for construction materials [23]. FWCNTs' outer walls can be connected to a composite's matrix, whereas the inner layers remain chemically intact and provide the high strength.

In this work, we examine the effect of the CNT water dispersion quality and the way of their preparation on the mechanical properties of cement pastes. We report that utilization of functionalized FWCNTs purified from CCFs resulted in 2-fold increase of the compressive strength. To the best of our knowledge, this is the highest value obtained using CNT water dispersion.

\section{Experimental Methods and Materials}

FWCNTs were synthesized by catalytic pyrolysis of methane at $960^{\circ} \mathrm{C}$ on the $\mathrm{Co}-\mathrm{Mo} / \mathrm{MgO}$ catalyst [24]. Briefly, a mixture of $\mathrm{Mg}\left(\mathrm{NO}_{3}\right)_{2} \cdot 6 \mathrm{H}_{2} \mathrm{O}(10.0 \mathrm{~g}), \mathrm{Co}\left(\mathrm{NO}_{3}\right)_{2} \cdot 6 \mathrm{H}_{2} \mathrm{O}(0.6 \mathrm{~g})$, $\left(\mathrm{NH}_{4}\right)_{6} \mathrm{Mo}_{7} \mathrm{O}_{24} \cdot 4 \mathrm{H}_{2} \mathrm{O}(0.073 \mathrm{~g}), \mathrm{NH}_{2} \mathrm{CH}_{2} \mathrm{COOH}(6.0 \mathrm{~g})$, and $\mathrm{H}_{2} \mathrm{O}(2.5 \mathrm{~mL})$ was carefully stirred to fully dissolve under heating conditions. Then, the cup containing the mixture was introduced for $20 \mathrm{~min}$ into a furnace heated to $550^{\circ} \mathrm{C}$ at ambient atmosphere to form highly porous $\mathrm{MgO}$ support with the Co-Mo catalyst. For the FWCNT synthesis a quartz boat with the freshly prepared catalyst (up to $4.0 \mathrm{~g}$ ) was placed in the center of the reactor. Then, the gas mixture of $\mathrm{CH}_{4}\left(150 \mathrm{~cm}^{3} / \mathrm{min}\right)$ and $\mathrm{H}_{2}\left(600 \mathrm{~cm}^{3} / \mathrm{min}\right)$ was introduced in the reactor for $10 \mathrm{~min}$. Then the reactor was heated up to $900^{\circ} \mathrm{C}$ at a rate of $30^{\circ} \mathrm{C} / \mathrm{min}$ for $1 \mathrm{~h}$ and then cooled down in an $\mathrm{H}_{2}$ flow.

Produced FWCNTs were purified with $\mathrm{HCl}$ (35 wt.\%) to remove residual catalyst and annealed at $380-390^{\circ} \mathrm{C}$ in an air atmosphere to eliminate amorphous carbon. The functionalization of FWCNTs was carried out in a mixture of nitric (70 wt.\%) and sulfuric (96wt.\%) acids with a volume ratio of $1: 3$ at $80^{\circ} \mathrm{C}$ for 3-4 hours under stirring conditions. Afterwards the tubes were washed by deionized water and $5 \%$
HCI until the sulfate ions were detectable in the infiltrated water via reaction with barium ions [25].

For the sample observations, the CNT aqueous dispersion was dropped on TEM grids, dried and observed in a double Cs-corrected JEOL JEM-2200FS transmission electron microscope (TEM) operated at an acceleration voltage of $200 \mathrm{kV}$. Investigations of cement hydration effect on the surface of CNTs were carried out by immerging TEM grids with CNTs into cement water solution for a certain period of time.

FT-IR spectra were measured from $\mathrm{KBr}$ pellets with the sample of CCFs at the concentration of around $1 \%$. The spectra were obtained using a Nicolet Magna 750 FT-IR spectrometer by averaging over 200 scans with a resolution of $2 \mathrm{~cm}^{-1}$.

The effect of CNT water dispersions was demonstrated by comparison of mechanical properties of cement paste beams prepared with as-functionalized FWCNTs containing CCFs (CCF/FWCNTs), functionalized FWCNTs purified from CCFs (CCF-free FWCNTs), and functionalized MWCNTs suspension with polyvinylpyrrolidone (PVP). PVP/MWCNT suspension (MWCNT diameter: $10 \div 20 \mathrm{~nm}$, purchased from Cheap Tubes Inc.) was prepared with the concentration of $0.12 \mathrm{wt} . \%$. In order to study the surfactant effect on mechanical properties we dispersed CCF/FWCNTs in 4\% water solution of sodium dodecyl sulfate (SDS). All CNT dispersions before testing were ultrasonicated using ultrasonic cell Branson $450-\mathrm{D}$ at $100 \mathrm{~W}$ and centrifuged at $2000 \mathrm{rpm}$ to remove CNT bundles.

In order to examine the effect of the presence of metal ions on the CNT sedimentation we utilized calcium $\left(2.96 \cdot 10^{-2}\right.$ wt.\%) and sodium $\left(2.58 \cdot 10^{-3}\right.$ wt.\%) hydroxides. Selected concentrations corresponded to the amount of $\mathrm{Ca}(\mathrm{OH})_{2}$ and $\mathrm{NaOH}$ during the cement hydration process [26]. The CNT concentration in each probe was $0.12 \mathrm{wt} . \%$.

For mechanical tests, we prepared cement paste blocks with the dimensions of $10 \times 10 \times 60 \mathrm{~mm}^{3}$. The ratio of water/cement was 0.4 by weight. The concentration of FWCNTs in cement paste was varied from 0.02 to $0.09 \mathrm{wt} . \%$. Three specimen beams were made for each probe.

\section{Experimental Results and Discussions}

TEM images confirmed the structure of FWCNTs with 2-5 walls in the diameter range from 4 to $10 \mathrm{~nm}$ (Figure $1(\mathrm{a})$ ). Figure 1 (b) shows a TEM image of FWCNTs covered by CCFs after acid functionalization (CCF/FWCNTs). In order to remove CCFs, the samples on the filter were iteratively washed by acetone until clean and colorless filtrate was obtained. Figure 1(c) shows functionalized FWCNTs after acetone washing procedure (CCF-free FWCNTs).

An FT-IR spectroscopy measurements were used to confirm the chemical nature of the compounds contained in the acetone washed waste (Figure 2). The peaks at 3600$3300 \mathrm{~cm}^{-1}$ are corresponded to $\mathrm{O}-\mathrm{H}$ stretching vibrations in alcohols, phenols, and -COOH. The peaks at 2924 and $2856 \mathrm{~cm}^{-1}$ are attributed to aliphatic $\mathrm{C}-\mathrm{H}$ stretches. The presence of the carboxyl groups is seen in the form of 


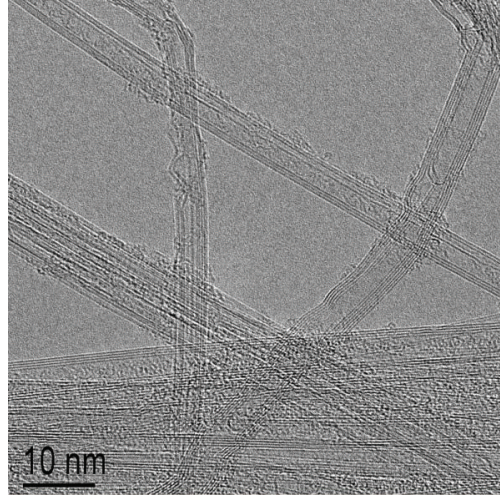

(a)

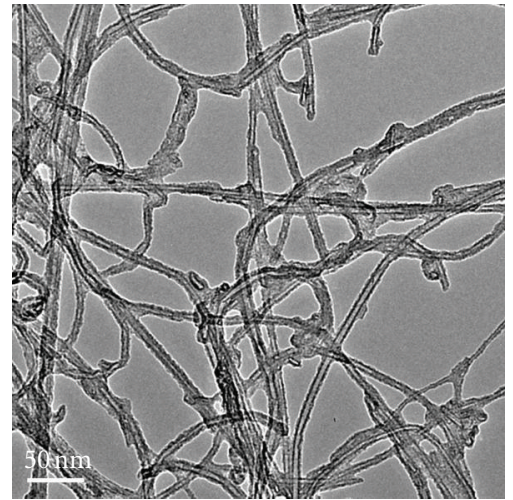

(b)

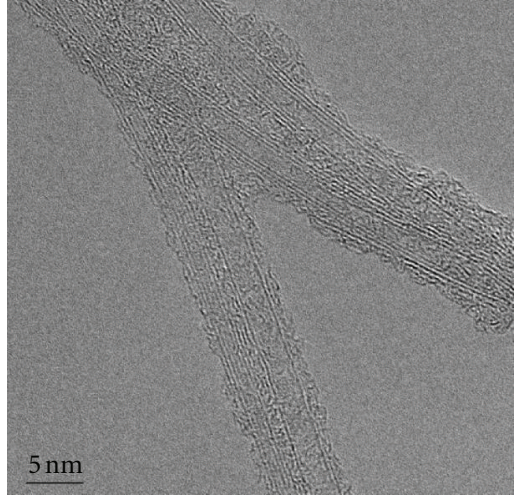

(c)

FIGURE 1: TEM images of different samples of carbon nanotubes: (a) as produced, (b) CCFs/FWCNTs, (c) and CCF-free FWCNTs.

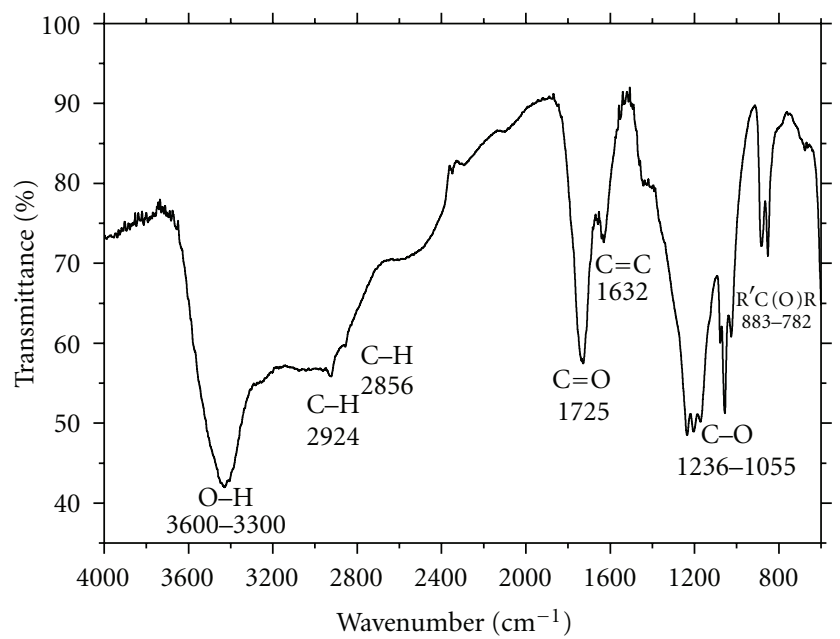

FIGURE 2: FT-IR spectrum of CCFs.

$\mathrm{C}=\mathrm{O}$ peaks $\left(1725 \mathrm{~cm}^{-1}\right), \mathrm{C}-\mathrm{O}$ stretches at $1236-1055 \mathrm{~cm}^{-1}$, and ketone at 883 and $782 \mathrm{~cm}^{-1}$. The peak at $1632 \mathrm{~cm}^{-1}$ can also be associated with aromatic $\mathrm{C}=\mathrm{C}$. These observations are consistent with proposals that the carboxylated carbonaceous fragments (CCFs) contain partially oxidized carbonaceous fragments terminated by a variety of oxygen containing groups.

Bond formation between functional groups on the surface of CNTs and cement hydration reaction product is very important to improve mechanical properties of cement composites. The functional groups $(-\mathrm{COOH}$ and $-\mathrm{OH})$ take part in reaction of ion exchange, replacing the proton by a metal ion. As it is known, cement grout consists of different types of metal ions, such as $\mathrm{Ca}^{2+}, \mathrm{Mg}^{2+}, \mathrm{Al}^{3+}, \mathrm{Fe}^{3+}, \mathrm{Na}^{+}$, and $\mathrm{K}^{+}$. It is worth noting that $\mathrm{Ca}^{2+}$ ions have the highest concentration in the mixture. Therefore, we examined the effect of $\mathrm{Ca}^{2+}$ ion presence on the stability of FWCNTs dispersions. For a comparison, we also investigated the effect of the presence of univalent $\mathrm{Na}^{+}$ions. Figure 3 shows the different CNT water dispersions. It can be seen that CCF-free FWCNTs form homogeneous water dispersion. Addition of $\mathrm{Ca}(\mathrm{OH})_{2}$

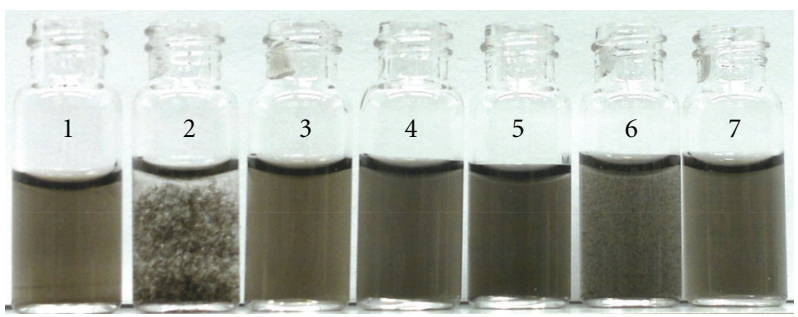

Figure 3: Aqueous CNT dispersion: (1) CCF-free FWCNTs; (2) CCF free FWCNTs $+\mathrm{Ca}(\mathrm{OH})_{2} ;$ (3) CCF-free FWCNTs + NaOH; (4) $\mathrm{PVP} / \mathrm{MWCNTs}+\mathrm{Ca}(\mathrm{OH})_{2}$; (5) PVP/MWCNTs $+\mathrm{NaOH}$; (6) $\mathrm{CCFs} / \mathrm{FWCNTs}+\mathrm{Ca}(\mathrm{OH})_{2} ;(7) \mathrm{CCFs} / \mathrm{FWCNTs}+\mathrm{NaOH}$. The CNT concentration in each probe is $0.12 \mathrm{wt} . \%$.

in the solution resulted in the floccules formation due to the reaction between functional groups on the surface of CNTs and $\mathrm{Ca}^{2+}$ ions, while introduction of univalent $\mathrm{Na}^{+}$ions did not practically affect the dispersion quality. The presence of $\mathrm{Ca}^{2+}$ ions also affected the dispersion of CCFs/FWCNTs; however, the floccules looked differently exhibiting the qualitative distinction with CCF-free FWCNTs. It can be also seen that the dispersion of PVP/MWCNTs was not affected by the presence of $\mathrm{Ca}^{2+}$ and $\mathrm{Na}^{+}$, conforming their inertness during the cement hydration process.

Concerning the effect of $\mathrm{NaOH}$ versus $\mathrm{Ca}(\mathrm{OH})_{2}$, one should mention that the presence of $\mathrm{Na}^{+}$ions did not lead to the precipitation of CNTs or deterioration of the dispersion quality. However, the precipitation of CNTs can be achieved when the ion concentration (or ionic strength) will be significantly higher $[27,28]$.

In order to confirm the possibility of cement hydration on the surface of CCF-free FWCNTs, we carried out microscopy investigations of the nanotubes placed on a TEM grid after 1 and 5.5 hours curing in water-cement solution $(1: 1)$. As can be seen from Figure 4(a), FWCNTs after acetone washing act as nucleation sites for precipitation of cement hydration products. 5.5-hour hydration sample shows the presence of C-S-H hydration products formed on the surface of FWCNTs. We believe that the present CCFs in 


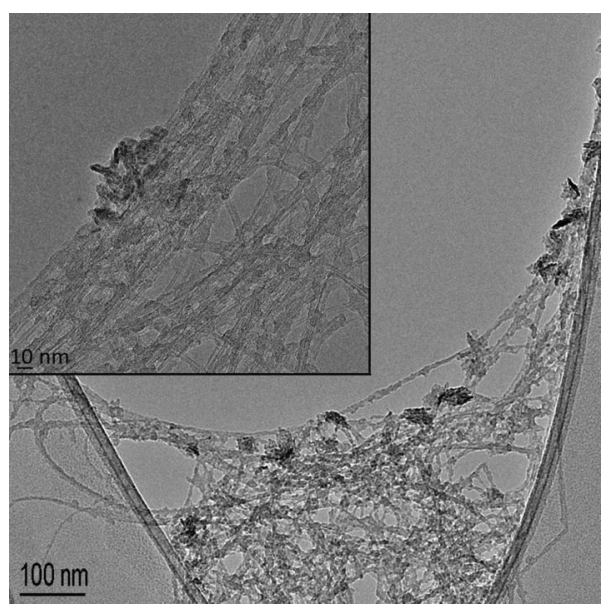

(a)

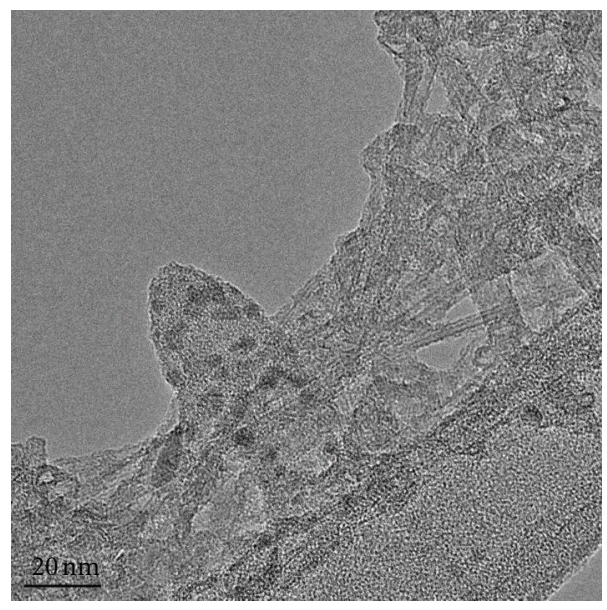

(b)

FIGURE 4: TEM images of different samples of CCF-free FWCNTs after cement hydration for (a) 1.0 and (b) 5.5 hours.

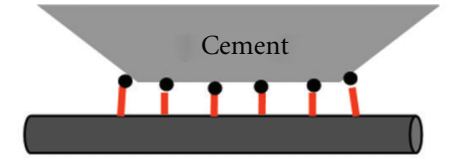

Functional groups
$\mathrm{Ca}^{2+}$ ions

(a)

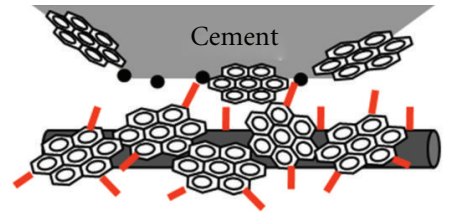

Functional groups

- $\mathrm{Ca}^{2+}$ ions

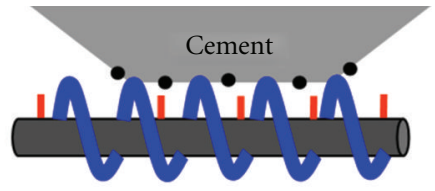

Functional groups $\bullet \mathrm{Ca}^{2+}$ ions Surfactant

(c)

FIGURE 5: A schematic representation of the reactivity of functionalized CNTs with cement hydration products: (a) CCF-free FWCNTs; (b) CCFs/FWCNTs; (c) surfactant-dispersed FWCNTs.

TABLE 1: Dependence of concentration FWCNTs on compressive strength of cement composite.

\begin{tabular}{cc}
\hline FWCNT concentration wt.\% & Compressive strength MPa \\
\hline- & 36 \\
0.02 & 66 \\
0.03 & 71 \\
0.05 & 65 \\
0.09 & 59 \\
0.09 & 59 \\
\hline
\end{tabular}

CCFs/FWCNTs can also act as nucleation sites for the cement hydration product formation, however without reinforcing effect and low bodings between CCFs and CNTs.

Good quality of the CNT water dispersions significantly affected the mechanical properties of the composite materials. Stable CCF-free FWCNTs water dispersions were added to Portland cement with the water/cement weight ratio of 0.3 . Table 1 summarized the results of the compressive strength measurements. As can be seen from the table, water dispersion with 0.03 wt.\% CCF-free FWCNT concentrations exhibited the best mechanical performance; the compressive strength is about two times higher than in the reference sample prepared without CNTs.

Similarly we investigated the effect of CCFs/FWCNTs on the compressive strength, but did not find such significant improvement. The dispersion with CCFs/FWCNTs ( 0.01 wt.\%) led to only $13 \%$ improvement in the compressive strength. This can be explained by the negative influence of CCFs.

Also we prepared a few samples of FWCNTs dispersed in water using SDS. The best concentration of the surfactant to prepare homogenous FWCNT water dispersion was found to be $4 \%$. The presence of surfactant resulted in the worsening of the mechanical properties of the cement composite material. The SDS sample showed 65\% decrease in the compressive strength.

Figure 5 summarizes our understanding of the effect of different dispersions on the chemical processes occurring during cement hydration. The best results can be obtained using CCF-free FWCNTs with - $\mathrm{COOH}$ and $-\mathrm{OH}$ functional groups attached to the outer side of the CNTs. In this case the CCF-free FWCNTs are chemically available for the reaction with hydration products: they can behave as nucleation sites and also can react with the hydration products forming 
interfacial mechanical bonds, while the performance of CCFs/FWCNTs is affected by the presence of CCFs in the dispersion and on the surface of CNTs. The CCFs are as reactive as functionalized CNTs but their presence does not lead to the mechanical reinforcement of the produced composite. Surfactant adsorbed on CNTs prevents the direct contact of the functional groups on the surface of FWCNTs and the cement substance.

\section{Conclusions}

We investigated the effect of the quality of carbon nanotube dispersions on the mechanical properties of cement pastes prepared using functionalized FWCNTs. The CNT functionalization was carried out in a mixture of concentrated $\mathrm{H}_{2} \mathrm{SO}_{4}$ and $\mathrm{HNO}_{3}$ at $80^{\circ} \mathrm{C}$. The functionalization acetone washing was found to be a very important procedure to remove carboxylated carbonaceous fragments that might negatively affect the mechanical strength due to their interaction with cement hydration product. There was found almost 2-fold increase in the compressive strength, when acetone washed FWCNTs were utilized. Utilisation of CCFs/FWCNTs led to only $13 \%$ improvement of the mechanical properties of the cement pastes. In spite of obtaining good CNT dispersions with the help of SDS, the compressive strength of the prepared paste decreased.

\section{Acknowledgments}

This work was supported by the Academy of Finland (project nos. 128445 and 124283) and TEKES (SIPI 211088).

\section{References}

[1] E. W. Wong, P. E. Sheehan, and C. M. Lieber, "Nanobeam mechanics: elasticity, strength, and toughness of nanorods and nanotubes," Science, vol. 277, no. 5334, pp. 1971-1975, 1997.

[2] G. Y. Li, P. M. Wang, and X. Zhao, "Mechanical behavior and microstructure of cement composites incorporating surfacetreated multi-walled carbon nanotubes," Carbon, vol. 43, no. 6, pp. 1239-1245, 2005.

[3] S. Musso, J. M. Tulliani, G. Ferro, and A. Tagliaferro, "Influence of carbon anotubes on the mechanical behavior of cement composites," Composites Science and Technology, vol. 69, no. 11-12, pp. 1985-1990, 2009.

[4] J. M. Makar, J. Margeson, and J. Luh, "Carbon nanotube/ cement composite-early results and potential application," in Proceedings of the 3rd International Conference on Constraction Materials: Perfomance, Innoations and Structural Implications, pp. 1-10, Vancouver, Canada, 2005.

[5] M. S. Konsta-Gdoutos, Z. S. Metaxa, and S. P. Shah, "Multiscale mechanical and fracture characteristics and early-age strain capacity of high performance carbon nanotube/cement nanocomposites," Cement and Concrete Composites, vol. 32, no. 2, pp. 110-115, 2010.

[6] A. Yazdanbakhsh, Z. Grasley, B. Tyson, and R. K. Abu Al-Rub, "Distribution of carbon nanofibers and nanotubes in cementitious composites," Transportation Research Record, no. 2142, pp. 89-95, 2010.
[7] M. S. Konsta-Gdoutos, Z. S. Metaxa, and S. P. Shah, "Highly dispersed carbon nanotube reinforced cement based materials," Cement and Concrete Research, vol. 40, no. 7, pp. 10521059, 2010.

[8] G. Y. Li, P. M. Wang, and X. Zhao, "Pressure-sensitive properties and microstructure of carbon nanotube reinforced cement composites," Cement and Concrete Composites, vol. 29, no. 5, pp. 377-382, 2007.

[9] J. Luo, Z. Duan, and H. Li, "The influence of surfactants on the processing of multi-walled carbon nanotubes in reinforced cement matrix composites," Physica Status Solidi A, vol. 206, no. 12, pp. 2783-2790, 2009.

[10] A. Cwirzen, K. Habermehl-Cwirzen, and V. Penttala, "Surface decoration of carbon nanotubes and mechanical properties of cement/carbon nanotube composites," Advances in Cement Research, vol. 20, no. 2, pp. 65-73, 2008.

[11] A. G. Nasibulin, S. D. Shandakov, L. I. Nasibulina et al., "A novel cement-based hybrid material," New Journal of Physics, vol. 11, Article ID 023013, 2009.

[12] L. I. Nasibulina, I. V. Anoshkin, S. D. Shandakov et al., "Direct synthesis of carbon nanofibers on cement particles," Transportation Research Record, no. 2142, pp. 96-101, 2010.

[13] L. I. Nasibulina, I. V. Anoshkin, A. V. Semencha et al., "Carbon nanofiber/clinker hybrid material as a highly efficient modificator of mortar mechanical properties," Materials Physics and Mechanics .In press.

[14] P. M. Ajayan, L. S. Schadler, C. Giannaris, and A. Rubio, "Single-walled carbon nanotube-polymer composites: strength and weakness," Advanced Materials, vol. 12, no. 10, pp. 750$753,2000$.

[15] Z. Wu, C. U. Pittman, and S. D. Gardner, "Nitric acid oxidation of carbon fibers and the effects of subsequent treatment in refluxing aqueous $\mathrm{NaOH}$," Carbon, vol. 33, no. 5, pp. 597-605, 1995.

[16] R. Verdejo, S. Lamoriniere, B. Cottam, A. Bismarck, and M. Shaffer, "Removal of oxidation debris from multi-walled carbon nanotubes," Chemical Communications, no. 5, pp. 513515, 2007.

[17] E. del Canto, K. Flavin, D. Movia, C. Navio, C. Bittencourt, and S. Giordani, "Critical investigation of defect site functionalization on single-walled carbon nanotubes," Chemistry of Materials, vol. 23, no. 1, pp. 67-74, 2011.

[18] R. Saito, G. Dresslhaus, and M. S. Dresselhaus, Physical Properties of Carbon Nanotubes, Imperial College Press, London, UK, 1996.

[19] M. Monthioux, B. W. Smith, B. Burteaux, A. Claye, J. E. Fischer, and D. E. Luzzi, "Sensitivity of single-wall carbon nanotubes to chemical processing: an electron microscopy investigation," Carbon, vol. 39, no. 8, pp. 1251-1272, 2001.

[20] X. Chen, X. Wang, and G. G. Sheng, "Effects of strain rates and temperatures on the mechanical properties of multi-walled carbon nanotubes," Physica Scripta, vol. 75, no. 4, pp. 455-459, 2007.

[21] B. Lukic, J. W. Seo, and R. R. Bacsa, "Catalytically grown carbon nanotubes of small diameter have a high young's modulus," Nano Letters, vol. 5, no. 10, pp. 2074-2077, 2005.

[22] Y. Hou, J. Tang, H. Zhang, C. Qian, Y. Feng, and J. Liu, "Functionalized few-walled carbon nanotubes for mechanical reinforcement of polymeric composites," ACS Nano, vol. 3, no. 5, pp. 1057-1062, 2009.

[23] T. Natsuki, K. Tantrakarn, and M. Endo, "Effects of carbon nanotube structures on mechanical properties," Applied Physics $A$, vol. 79, no. 1, pp. 117-124, 2004. 
[24] E. G. Rakov, D. A. Grishin, Y. V. Gavrilov et al., "The morphology of pyrolytic carbon nanotubes with a small number of walls," Russian Journal of Physical Chemistry A, vol. 78, no. 12, pp. 1966-1971, 2004.

[25] N. T. Hung, I. V. Anoshkin, A. P. Dementjev, D. V. Katorov, and E. G. Rakov, "Functionalization and solubilization of thin multiwalled carbon nanotubes," Inorganic Materials, vol. 44, no. 3, pp. 219-223, 2008.

[26] P. C. Hewlett, Lea's Chemistry of Cement and Concrete, Arnold, London, UK, 4th edition, 1998.

[27] X. Peng, J. Jia, X. Gong, Z. Luan, and B. Fan, "Aqueous stability of oxidized carbon nanotubes and the precipitation by salts," Journal of Hazardous Materials, vol. 165, no. 1-3, pp. 12391242, 2009.

[28] B. Smith, K. Wepasnick, K. E. Schrote et al., "Colloidal properties of aqueous suspensions of acid-treated, multi-walled carbon nanotubes," Environmental Science and Technology, vol. 43, no. 3, pp. 819-825, 2009. 

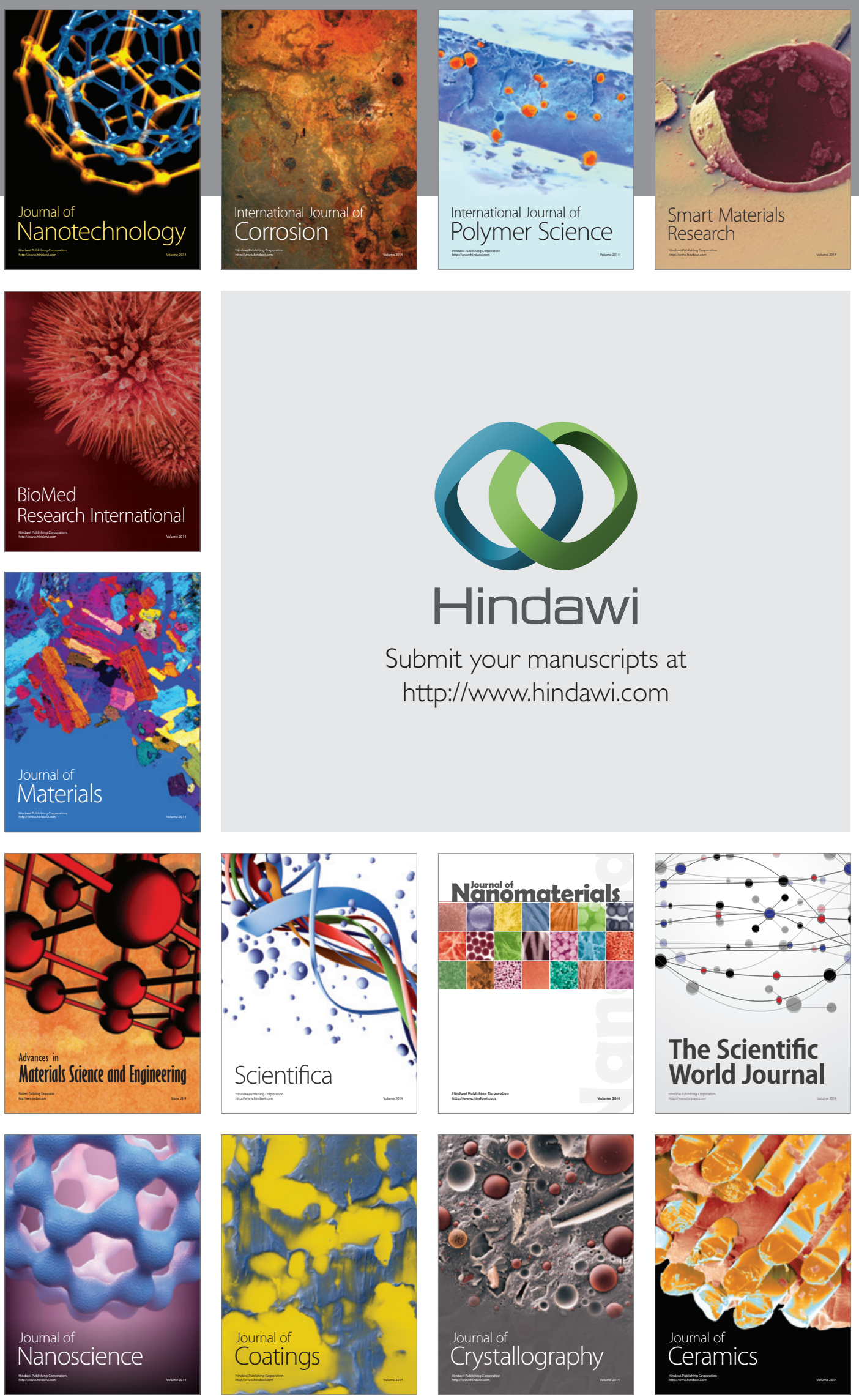

The Scientific World Journal

Submit your manuscripts at

http://www.hindawi.com

\section{World Journal}

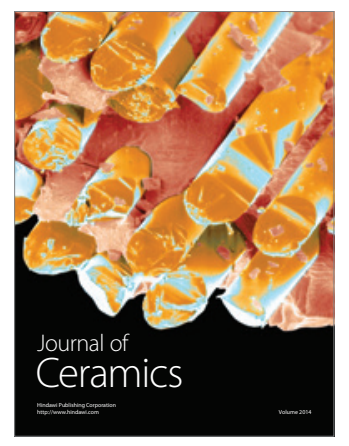

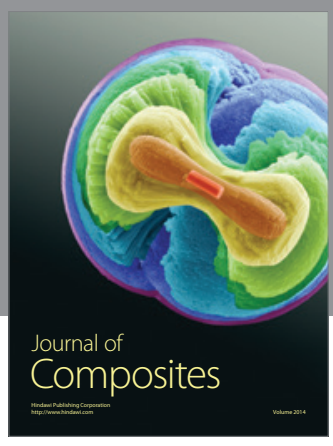
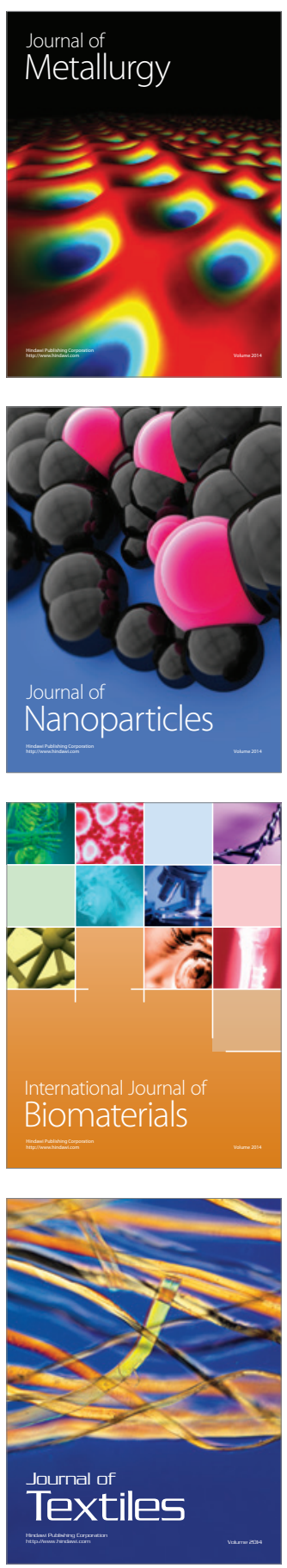